\title{
Entretien avec Cécile Boulaire
}

\section{Christine Boutevin et Frédéric Torterat}

\author{
(2) OpenEdition \\ Journals \\ Édition électronique \\ URL : https://journals.openedition.org/trema/6479 \\ DOI : $10.4000 /$ trema.6479 \\ ISSN : 2107-0997 \\ Éditeur \\ Faculté d'Éducation de l'université de Montpellier
}

Référence électronique

Christine Boutevin et Frédéric Torterat, «Entretien avec Cécile Boulaire », Tréma [En ligne], 55 | 2021, mis en ligne le 20 octobre 2021, consulté le 14 décembre 2021. URL : http://journals.openedition.org/ trema/6479; DOI : https://doi.org/10.4000/trema.6479

Ce document a été généré automatiquement le 14 décembre 2021.

Trema 


\title{
Entretien avec Cécile Boulaire
}

\author{
Christine Boutevin et Frédéric Torterat
}

Vous êtes à l'initiative d'un projet, Lire aux bébés, avec à l'origine votre engagement dans une association. Pouvez-vous nous présenter brièvement le projet?

1 Le CHU de Tours a ouvert son nouveau service de néonatologie en 2018, transférant le soin des bébés prématurés, auparavant accueillis dans un hôpital pédiatrique aux équipements désuets, au plus près du Centre Olympe de Gouges dédié à la gynécologie et à la maternité. Au sein de ce service de pointe, les parents peuvent vivre auprès de leur enfant prématuré pendant toute la durée de son hospitalisation en service de réanimation, de soins intensifs, puis de soins de suite, jusqu'au moment où l'enfant est apte à rejoindre le foyer familial. Le projet du service a en effet été centré sur la parentalité.

Or pendant toute la durée de cette hospitalisation, pendant laquelle le bébé bénéficie de soins hospitaliers d'une grande technicité, il importe aussi qu'il puisse se construire en tant que petit humain, enfant de ses parents ; les parents, quant à eux, doivent trouver la place nécessaire pour construire leur rapport parental à ce bébé, alors même qu'ils ne vivent pas chez eux, qu'ils doivent parfois s'occuper du reste de la fratrie, qu'il faut composer avec l'environnement hospitalier, les soins médicaux et la présence de nombreux soignants.

La construction de la parentalité est freinée par de nombreux facteurs :

- Le fait de ne pas être « chez soi », et souvent, le souci de la fratrie

- L'inquiétude des parents quant à la santé de l'enfant

- La culpabilité de la mère (de ne pas avoir pu mener la grossesse à son terme; de ne pas pouvoir « nourrir » son nouveau-né ; de ne pas savoir comment apaiser ses souffrances)

- Le sentiment de «non-compétence », comparativement aux équipes soignantes

- L'isolement de la mère par rapport à son milieu culturel

Ces facteurs produisent chez les parents une forme de sidération, qui freine notamment le développement d'une parole naturelle adressée à l'enfant. Or il est fondamental pour le bon développement général de l'enfant (psychique, affectif, cognitif) qu'il puisse bénéficier, malgré sa prématurité, du «maternage » actif qui lui permet d'exister en tant que sujet. À cet égard, tout ce qui, au sein des services de néonatologie, favorise la construction du lien parent-enfant, et en particulier mère-enfant, améliore le 
développement de l'enfant. Dans ce domaine, comme on le sait, la parole joue un rôle crucial (Filippa, Kuhn \& Westrup 2017).

3 J'ai rencontré, dans le cadre de mes enseignements au sein de l'école d'orthophonie de la faculté de médecine, lors d'une soutenance de mémoire, l'orthophoniste de ce service. Nous avions pu nous rendre compte lors de cette soutenance que nous partagions beaucoup de points de vue sur le livre pour enfants. A cette occasion, elle m'avait expliqué son travail en néonatalogie, dont j'ignorais tout, qui consistait non pas à aborder la question du langage mais l'apprentissage de la déglutition, du placement de la langue car les bébés prématurés ne savent pas téter. Au fil de la conversation, elle m'a fait part de l'inquiétude du personnel soignant autour de ce que j'appellerais une " parole empêchée ». En effet force était de constater par ces soignants que les parents, malgré un environnement adapté, ne restaient pas auprès de leur bébé autant que l'équipe soignante l'aurait souhaité, malgré la possibilité de loger sur place, et évoquaient, quand ils le pouvaient, qu'ils ne savaient pas quoi dire à ce tout-petit très appareillé, parfois en grande souffrance. Tout cela pouvait les amener à rester «sans voix » et même à tarder à créer le lien à cause de la peur de perdre à cet enfant. Tous les contacts non verbaux et verbaux qui se font lors de l'arrivée à la maison d'un nouveau-né n'avaient pas lieu, ce qui inquiétait beaucoup le personnel de néonatologie.

C'est ensemble, et avec l'association Livre passerelle dès le départ, que nous avons eu l'idée de "prêter les mots des albums" aux familles, le temps de l'hospitalisation de leur bébé. Ce projet s'appuie sur la conviction qu'intégrer au sein du service de néonatologie une pratique active, par les équipes, de cette première culture orale de l'enfance (berceuses et comptines, jeux chantés, albums pour le premier âge), contribuerait à établir, autour des dyades parent-enfant, une atmosphère de parentalité à la fois plus "naturelle » (au sens de «banale, ordinaire, familière ») et plus «culturelle» (faisant entrer le petit enfant dans une communauté de partage, à travers le plaisir de l'écoute), contrebalançant la dimension très technique de l'établissement. On fait le pari que les pratiques développées par les lectrices et reprises par les soignants inviteraient les parents, de manière plus ou moins incitative, à engager eux-mêmes une pratique de chant, jeu chanté, comptine, partage d'albums avec leur enfant, le livre étant conçu comme un intermédiaire pour déclencher cette parole jusque-là empêchée.

5 L'une des hypothèses de ce projet, étayée par plusieurs décennies d'actions comparables menées au sein de l'association d'éducation populaire Livre passerelle, partie prenante du projet depuis le début, est notamment que l'album de littérature pour la jeunesse, s'il est judicieusement choisi en fonction de sa qualité poétique et symbolique, "prête » aux parents les mots et les rythmes permettant de s'adresser à leur bébé, notamment dans un contexte où le parent peine à trouver le registre sur lequel partager, par le langage, un univers d'images, de promesses, de valeurs et d'imaginaire enfantin. Plus que les propos ordinaires des échanges quotidiens, les mots des livres disent notre humanité : ils sont alors une passerelle entre les adultes et les bébés, exprimant l'émotion et la tendresse des adultes, construisant la confiance des bébés.

Comment conciliez-vous cette dimension militante avec un questionnement scientifique? En quoi ces deux aspects du travail se nourrissent-ils mutuellement selon vous ?

6 Il n'y a pas de séparation entre un engagement associatif et une activité de recherche scientifique, dans mon cas. En effet, mon entrée à l'association Livre passerelle coïncide 
à peu près avec mon entrée à l'université comme maitre de conférences : ce sont deux espaces au sein desquels, de manière complémentaire, développer une réflexion autour de l'album pour enfants et de son rôle dans la construction des enfants. À l'université, cela se fait par la réflexion, la construction de ressources intellectuelles, la confrontation avec d'autres travaux de recherches, issus d'autres disciplines que la mienne, et par l'enseignement, qui est toujours une manière de tester ses réflexions, en les confrontant aux préjugés infondés mais aussi objections constructives que peuvent formuler, en groupe, les étudiants. Mon rôle dans l'association Livre passerelle a longtemps consisté en sessions de formation, mais celles-ci, adressées à des adultes et non à des jeunes, étaient l'occasion de confronter mes enseignements à des pratiques et des cultures professionnelles déjà construites. Aujourd'hui, au sein du projet «Lire aux bébés / Lisons aux nourrissons ", mon rôle consiste aussi à lire directement, et cette pratique change radicalement mon regard sur les interactions entre livres, bébés et adultes (parents et soignants).

7 En attendant la fin de la crise sanitaire qui a contraint notre équipe à repousser nos expérimentations, nous sommes intervenus dans une unité "parent-bébé » de suivi psychiatrique périnatal. Dans ce cadre, notre équipe participe aussi à des moments de vie très particuliers : ainsi avons-nous pu accompagner une mère accueillie dans une unité périnatale avec son enfant, tous deux en grande détresse. Pendant des semaines, cette jeune maman n'a pas voulu qu'on lui lise une histoire : elle plaçait entre elle et le monde extérieur le berceau où se trouvait son tout petit - en marge de nos interventions lecture, elle avait confié aux soignants ne jamais avoir entendu d'histoire dans sa propre enfance. Notre pratique de lecture orale d'albums semblait lui faire violence : elle ne comprenait pas ce qu'on attendait, elle ne savait probablement pas comment elle devait réagir. Au bout de quelques semaines, elle a consenti à ce qu'on lui raconte une histoire, à elle et son bébé, et la dernière fois qu'on l'a vue, après trois mois d'interventions à raison d'une par semaine et autant d'occasions où elle nous a vu faire, c'est elle qui racontait à son bébé.

8 L'association est une instance qui agit concrètement dans le milieu social : elle est le prolongement logique de mon activité d'enseignement et de recherche, le but vers lequel tendent mes réflexions intellectuelles et mes enseignements. Comment appuyer les intuitions développées sur le terrain dans le cadre associatif, par des recherches visant à construire les modèles abstraits qui fondent ces intuitions en raison? Comment interroger de manière critique certaines pratiques, ancrées dans la vie de l'association, en les confrontant aux recherches les plus actuelles, qui peuvent parfois invalider ce qu'on croyait acquis, ou proposer un regard nouveau sur un fonctionnement qu'on avait initialement analysé à la lumière d'un autre corpus critique ? Il s'agit de constants allers-retours entre recherche théorique, enseignement, pratique de terrain.

Quelles sont les spécificités pour vous, notamment comme chercheuse en littérature, du travail avec des professionnels du monde médical?

9 Lorsqu'on vient des études littéraires, on "n'existe " même pas aux yeux de la communauté des sciences dites « dures ", qui considère, sans doute avec des éléments tangibles, qu'on ne «fait pas de recherche ». Mais le cas est légèrement différent avec les spécialistes des sciences médicales. En effet, pour eux, la recherche se divise en deux branches, la recherche fondamentale, et la recherche clinique. C'est plutôt sous ce deuxième angle qu'ils peuvent éventuellement manifester un certain intérêt pour ce 
qu'on peut apporter, car il s'agit d'agir "sur le terrain ", auprès de vraies personnes et que, dans ce contexte, la question du rapport individuel au sens refait surface, celle précisément qui me paraît l'un des éléments centraux des études littéraires.

Travailler dans le monde médical suppose de se décentrer, et de faire preuve de beaucoup de modestie - je trouve que c'est toujours sain, dans les deux cas! Ces professionnels ne connaissent rien de mon corpus de références intellectuelles : je dois donc m'en faire, en quelque sorte, la «traductrice », la médiatrice. Cela suppose que je sache faire un tri drastique entre les références que j'utilise par commodité, par habitude, par paresse intellectuelle, parce qu'ils «font partie des murs » dans ma discipline ici et maintenant, et les références auxquelles je crois profondément, parce qu'elles ont fini par constituer, au fil des mises à l'épreuve, un bagage intellectuel qui m'aide à approfondir ce que je crois être notre rapport à la littérature. Ce contact avec des chercheurs et professionnels étrangers à ma discipline m'a aidée à "élaguer " considérablement: je ne m'embarrasse plus des travaux abscons, de certaines références que je traînais par une sotte allégeance à mes maîtres ; il me semble parfois aussi que je vois plus clair dans le "champ » nébuleux qui constitue la réflexion littéraire : je détecte les propos ou les livres qui ne sont écrits que pour occuper une position dans le champ, mais qui n'apportent en réalité aucune contribution réelle et constructive à la construction d'une pensée de la littérature. Ces propos peuvent paraître totalement outrecuidants! Mais il est vrai que la nécessité de parler avec des acteurs très éloignés du milieu académique littéraire m'a conduite à une grande exigence dans mes références, j'ai développé un regard plus critique, parce que plus concerné. Traduire suppose de comprendre en profondeur, et on fait vite le tri entre ce qu'on veut partager, et ce qui ne mérite pas de l'être.

Comment votre expertise de la littérature de jeunesse intervient-elle dans cette expérimentation?

11 Nous travaillons avec des professionnels dont la spécialité consiste à faire survivre de très petits bébés, ou à arracher des dyades parent-bébé à la détresse de la dépression ou de la maladie psychique. Leurs compétences sont extrêmement pointues dans leurs divers domaines. Sur le plan des lectures adressées à des enfants, en revanche, ils se situent exactement dans la moyenne des représentations. Si j'exagère un peu, je peux dire que les représentations que ces professionnels se font du livre pour enfants, de ce qu'il devrait être, et de ce à quoi il sert, ne sont guère éloignées de celles que j'observe auprès des étudiants de première année que j'accueille tous les ans en septembre dans mes cours. Je sais, donc, assez précisément, ce qu'ils pensent des livres et des manières de les partager. Mon expertise - mais aussi celle des professionnelles de Livre passerelle - consiste à les amener tout doucement, par l'exemple, par le plaisir partagé, à découvrir que le livre peut être autre. Que des histoires pour très petits enfants peuvent être profondes et belles, qu'elles peuvent nous toucher, nous adultes, au plus profond de quelque chose. Qu'elles méritent une attention délicate au moment de leur "performance ", comme un poème, parce que ce ne sont pas des suppositoires pour endormir les enfants, mais des œuvres qui ont été composées avec soin pour parler véritablement au bébé, du bébé, avec le bébé. C'est pour cette raison que la littérature est un très bon auxiliaire pour permettre, à une maman, d'entrer dans un échange langagier tout à fait banal avec son bébé.

12 Mais il faut dire que ce projet a changé mon regard sur les livres pour enfants. J'étais extrêmement sensible à la dimension plastique de ces ouvrages, dimension que j'ai 
ardemment défendue jusqu'à dire qu'il s'agissait d'un élément littéraire. Aujourd'hui je me rends compte que ces albums ne sont pas toujours satisfaisants soit sur le plan narratif pour le double lecteur parent et bébé, soit sur le plan poétique qui fait que l'on peut lire pendant des semaines le même livre avec un plaisir toujours intact. Corinne Dreyfuss, par exemple dans Pomme Pomme Pomme a ce talent, cet art d'écrire pour les enfants, comme l'avait Margaret Wise Brown, dans Bonsoir lune, pour trouver la langue qui plaît aux bébés.

Comment envisagez-vous d'impliquer les parents de ces tout-petits ?

13 Le cœur du projet est en réalité le parent, pas le bébé. Nous ne venons pas en néonatologie pour faire plaisir aux petits. Cela, les associations comme les «blouses roses » ou le « rire médecine » le font dans les hôpitaux pédiatriques. Notre action se situe légèrement à côté : nous venons pour travailler sur le lien. Les travaux les plus récents ont montré l'importance de la parole adressée au bébé prématuré, et plus encore, la parole maternelle - et, au sein des propos adressés par la mère, on a pu montrer que la parole libre était plus intéressante que la lecture à haute voix. Notons que certaines mères ne s'autorisent pas à faire ces lectures, notamment parce qu'elles ne parlent pas le français ou depuis peu, ayant le sentiment qu'elles ne pourraient pas interagir, ce qui pour nous n'est pourtant pas un problème, dans la mesure où le Livre passerelle intervient fréquemment en PMI avec des familles non francophones. Or, dans ce contexte-là comme dans d'autres, le fait de ne pas être francophone peut constituer un facteur de vulnérabilité. À cet égard, on sent très fort le poids des déterminations sociales. Notre rôle est donc bien modeste : nous ne sommes pas là pour construire le lien entre nous et les bébés, nous sommes là pour déclencher la construction du lien entre les parents sidérés et leur bébé. Toute la délicatesse de cette action consiste donc à nous adresser au bébé en présence de ses parents - en présence aussi des soignants, dont nous savons que, par imprégnation, ils se feront le relais de ces lectures s'ils les jugent bénéfiques. C'est d'abord le parent que nous cherchons à émerveiller, en lui montrant que son enfant est sensible à l'histoire qui lui est offerte, qu'il réagit, qu'il « comprend » - pas l'histoire elle-même, bien sûr, mais il comprend qu'on s'adresse à lui, que ce n'est pas la parole ordinaire qui lui est offerte à ce moment-là, et que c'est un moment spécial qu'il partage avec la maman qui le tient contre elle. Nous cherchons, en accord avec les équipes soignantes, à offrir aux parents un autre regard sur leur bébé : ce petit battant, ce champion de la survie, pour une fois on vient lui offrir un moment qui n'est pas un soin, et qui est fait de langage, partagé pour le plaisir. C'est la décharge émotionnelle de ce moment partagé qui va toucher le parent, et nous faisons le pari que, pour revivre l'intensité de ce moment, les parents à leur tour s'empareront des livres, et que cette parole "empruntée " ouvrira sur d'autres paroles, libres, adressées naturellement aux bébés, par-delà l'anxiété ou la lassitude du milieu hospitalier. Les études sont claires : les bébés qui bénéficient d'une stimulation parentale, principalement langagière, compensent plus vite les difficultés d'organisation neuronale liée à la prématurité. Nous voulons déclencher cet échange langagier affectueux entre les parents et leur bébé, et pour cela, c'est d'abord eux que nous voulons toucher, émouvoir.

Vous avez régulièrement l'occasion d'échanger sur ces questions avec des linguistes au sein des séminaires de recherche, mais également avec des orthophonistes: comment 
ressentez-vous aujourd'hui l'importance du langage dans la construction du lien avec l'enfant?

14 Pour moi la relation à l'enfant par le langage est le cœur de tout. J'ai eu l'occasion de m'exprimer, ces dernières années, sur des programmes de stimulation langagière qui, à mes yeux, manquaient partiellement leur but, parce qu'ils se focalisaient sur la dimension quantitative (combien de mots acquis ?) alors que leur cœur de la question, c'est la relation: non pas «comment parlons-nous? «(riche, pauvre, avec peu ou beaucoup de vocabulaire) mais " pour quelle raison parlons-nous? ». Un enfant vient à parler parce qu'on lui parle, et parce qu'en lui parlant on le fait exister comme sujet. René Diatkine, à la suite de Winnicott, dit que c'est parce qu'il peut se faire une représentation symbolique de sa mère que le bébé peut survivre, psychiquement, aux moments où il prend conscience qu'elle le laisse : et rien n'est plus symbolique que le mot - parce qu'il peut être partagé. Le bébé ne peut partager avec personne son image mentale de sa mère, ni son odeur (qui imprègne peut-être encore le doudou qu'il serre contre lui), mais le mot "maman" peut lui être dit et redit par la nourrice ou la puéricultrice qui prennent soin de lui, et rappellent à sa conscience que l'absence qui le ronge a un nom, et que ce nom est relié à une réalité. En ce sens, le langage est vital, il permet au sujet d'exister, et il lui permet de faire lien avec l'autre.

Mais parfois, la parole est embarrassée, empêchée. Nous travaillons actuellement, avec l'association Livre passerelle, dans une structure qui a bien voulu accueillir le programme "Lire aux bébés » alors que la situation sanitaire nous interdisait encore l'accès à la néonatologie. Il s'agit d'une unité périnatale qui accueille des mères et leur bébé, parfois aussi des pères, dans un contexte de fragilisation du lien juste après la naissance (souvent, une décompensation psychique). Ces parents sont assaillis par de graves angoisses à l'occasion de cette naissance, qui fait parfois resurgir des problèmes psychiques anciens, et qui pose aussi certaines fois de terribles problèmes matériels intriqués aux problèmes affectifs. Nous sommes frappées de voir que ce nœud d'angoisses sédimentées, qui explose autour de la naissance de cet enfant, coupe littéralement la parole aux parents. Notre action est très modeste, nous intervenons une matinée par semaine et nous lisons, dans la salle commune, des histoires aux bébés et à leurs parents. Et nous percevons immédiatement le soupir de satisfaction des soignants quand, au bout de nombreuses semaines, une maman s'empare à son tour d'un petit album cartonné, le lit à son bébé, et poursuit sa lecture en lui adressant, à lui, quelques mots de commentaires qu'elle puise dans la force de sa relation à son enfant. De notre côté, l'on recherche des textes où le récit est juste, les émotions sont claires, avec pour objectif de raconter des histoires qui vont «sonner » à l'oreille des enfants, qui vont avoir cette belle qualité poétique et rythmique contribuant au fait que les enfants saisissent que quelque chose de singulier est en train de se dire, même s'ils n'en comprennent pas forcément la sémantique. Nous n'avons pas d'exigence particulière en termes linguistiques, par exemple par rapport aux temps verbaux ou aux marques du dialogue, mais ce qui nous intéresse avant tout, ce sont des albums qui touchent simultanément nos deux publics, parents et enfants.

Bien sûr, ce ne sont pas ces lectures seules qui provoquent cette libération! C'est bien parce que ces mères sont accueillies dans une structure psychiatrique ouverte, entourées par des professionnelles d'horizons divers, qu'elles parviennent à faire leur chemin, dans leur vie et vers leur bébé. Cela n'est évidemment pas comparable avec la lecture scolaire, qui fait face à d'autres impératifs, pédagogiques et didactiques. Mais nous observons que le livre est un déclencheur de parole, lorsqu'il est bien choisi, 
lorsqu'il est partagé avec une authentique sensibilité à son pouvoir poétique. Pour revenir au langage : il me semble absolument central dans le développement de l'enfant - même si on peut aussi, bien entendu, s'intéresser aux autres formes du maternage, au portage, etc. Mais le maternage et le portage concernent la relation du bébé à sa mère, tandis que le langage concerne la relation qu'il aura avec toutes les autres personnes de son entourage.

\section{BIBLIOGRAPHIE}

Filippa, Manuela ; Kuhn, Pierre \& Westrup Bjorn (éd.) (2017). Early Vocal Contact and Preterm Infant Brain Development. Bridging the Gaps Between Research and Practice. London : Springer. 